Research Paper

\title{
Loss of LKBI Expression Decreases the Survival and Promotes Laryngeal Cancer Metastasis
}

\author{
Sha-sha He ${ }^{1,3 *}$, Yong Chen1,3*, Hong-zhi Wang7* , Xiao-ming Shen"1, 6, Peng Sun 1, 5, Jun Dong1, 2, Xin-biao \\ Liao $^{8}$, Gui-fang Guo ${ }^{1,2}$, Ju-gao Chen ${ }^{1}$, Liang-ping Xia1, 2, Pei-li Hu1, 2, Hui-juan Qiu1,2, Shou-sheng Liu1, 2 ,

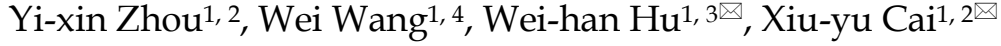 \\ 1. State Key Laboratory of Oncology in South China, Cancer Center, Sun Yat-Sen University, Guangzhou; \\ 2. Department of VIP Region, Cancer Center, Sun Yat-Sen University, Guangzhou, China; \\ 3. Department of Radiation, Cancer Center, Sun Yat-Sen University, Guangzhou, China; \\ 4. Department of Gastric Surgery, Cancer Center, Sun Yat-Sen University, Guangzhou, China; \\ 5. Department of Pathology, Cancer Center, Sun Yat-Sen University, Guangzhou, China: \\ 6. Department of Radiology, The First People's Hospital of Foshan (The affiliated Foshan Hospital of Sun Yat-Sen University), Foshan, Guangdong, China; \\ 7. Department of Radiation Oncology, Cancer Hospital, Chinese Academy of Medical Science, Peking Union Medical College, Beijing 100021, China; \\ 8. Department of Radiation Oncology, The First Affiliated Hospital of Zhejiang University, Hangzhou, China. \\ * These authors have contributed equally to this work
}

$\triangle$ Corresponding authors: Wei-han Hu M.D., Ph.D. State Key Laboratory of Oncology in South China \& Department of Medical Oncology, Sun Yat-sen University Cancer Center, Guangzhou, 510060, China. Telephone: +0086-020-87343505, E-mail: huwh@sysucc.org.cn Xiu-yu Cai M.D., Ph.D. State Key Laboratory of Oncology in South China \& Department of VIP Region, Sun Yat-sen University Cancer Center, Guangzhou, 510060, China. Telephone: +0086-020-87343498, E-mail: caixy@sysucc.org.cn

(1) Ivyspring International Publisher. This is an open access article distributed under the terms of the Creative Commons Attribution (CC BY-NC) license (https:// creativecommons.org/licenses/by-nc/4.0/). See http://ivyspring.com/terms for full terms and conditions.

Received: 2017.04.12; Accepted: 2017.09.07; Published: 2017.09.30

\begin{abstract}
Background: Given recent results indicating that diminished LKB1 expression in laryngeal cancer correlates with shorter survival. We aim to perform an analysis estimate the role of decreased liver kinase $\mathrm{Bl}(\mathrm{LKBl})$ and in the prognostication of human laryngeal squamous cell carcinoma (LSCC).

Methods: We conducted a retrospective study and evaluate the expression of LKBI and p16INK4a (p16) in 208 clinical advanced-stage LSCC tissue samples by using immunohistochemistry. The specimens were received at Sun Yat-sen University Cancer Center (Guangzhou, China). To evaluate the independent prognostic relevance of LKBI, univariate and multivariate Cox regression models were used, overall survival (OS) and distant metastasis-free survival (DMFS) were compared using the Kaplan-Meier method.

Results: Immunohistochemical analyses revealed that 80/208 (38.5\%) of the LSCC tissue samples expressed high LKBI. Low LKBI expression was associated with a significantly shorter OS and DMFS than high LKB1 expression $(P=0.041$ and 0.028 , respectively; log-rank test), and there was a poorer OS in the p16-positive than p16-negative group. In the subgroup stratified by p16 status, the shorter OS were also seen with low LKBI expression. Multivariate survival analysis indicated that high LKBI expression was an independent prognostic factor for OS (hazard ratio [HR]: 1.628, $95 \%$ confidence interval $[\mathrm{Cl}]: 1.060-2.500, P=0.026)$ and DMFS (HR: 2.182, 95\% Cl: 1.069-4.456, $P=0.032$ ).

Conclusions: Our data indicated that low expression of LKBI was significantly associated with poor prognosis and it may represent a marker of tumor metastasis in patients with LSCC. When combined with p16, LKBI was also of prognostic value.
\end{abstract}

Key words: Laryngeal squamous cell carcinoma; liver kinase B1 (LKB1); p16INK4a (p16); prognosis. 


\section{Introduction}

Liver kinase B1 (LKB1) is a protein kinase, also known as serine/threonine kinase 11 (STK11) gene [1], which has been commonly identified as a critical cancer suppressor in many cancer cells. LKB1 is the key protein responsible for tumor suppression, cell growth regulation, and apoptosis promotion [2]. It is identified that LKB1 as a gene is responsible for the Peutz-Jeghers Syndrome (PJS), which displays an increased predisposition to malignant tumors in multiple tissues. PJS is an autosomal dominant disorder characterized by benign polyps in the gastrointestinal tract $[3,4]$. Mutation of LKB1 usually be found in patients with PJS [5]. LKB1 may serves as a metabolic checkpoint to coordinate cell growth and survival with energy metabolism [6]. LKB1 deficiency induces the metabolic remodelling of energy through the downstream effectors of AMP-dependent protein kinase (AMPK), which is as a partner in cellular energy sensor of LKB1 [7, 8]. AMPK was phosphorylated by LKB1 when the AMP/ATP ratio is high [9], and plays vital roles in the maintenance of cell polarity and inhibits the inappropriate expansion of cancer cells, although they really have different roles in carcinogenesis. Cancer-associated epithelial-mesenchymal transition (EMT), which also influences cell metabolism is involved in epigenetics and differentiation of cancer, through which differentiate depithelial cancer cells reverse to an undifferentiated state $[10,11]$. This reverse transition plays a key role in the formation of macroscopic metastases in different organs [12]. A study [10] found that anti-EMT therapies have great potential to reverse cancer cell dedifferentiation and retain them in a more differentiated epithelial state, even bring good opportunities to overcome resistance to conventional cancer treatments and inhibit cancer metastasis.

Similarly, studies have demonstrated that low LKB1 protein expression is associated with worse overall survival (OS) in human cancers, such as colorectal cancer [13], lung cancer [14], breast cancer [15], hepatocellular carcinoma [16], and head and neck squamous cell carcinoma [17]. A meta-analysis [18] have revealed that decreased expression of LKB1 protein was significantly associated with OS in relation to solid tumors (HR: 1.86, 95\%CI: 1.42-2.42, $P<0.001$ ), but not for disease-free survival (DFS). In breast cancer, there is a discovery that $10 \%$ of metastases in hematopoietic system showed loss of LKB1 expression [19]. Zhuang et al. [21] have demonstrated that overexpression of LKB1 protein reduces breast cancer microvessel density and inhibits metastasis [20].
p16INK4a (p16), a cyclin-dependent kinase inhibitor that prevents retinoblastoma phosphorylation and blocks cell-cycle progression at the G1-S checkpoint, is involved in head and neck squamous cell carcinoma (HNSCC) pathogenesis [21]. It has found that p16 sometimes represent as a surrogate biomarker for HPV-associated HNSCC and can also indicate the prognosis [22].

Although the fact that the mechanism by which LKB1 acts as a tumour suppressor has yet been unclear and even some conflicting results have also been reported. Consequently, we initiated a study to evaluate the significance of decreased LKB1 expression and in combination of p16 status to predict clinical outcomes in laryngeal cancer by using the immunohistochemistry (IHC) test method.

\section{Methods}

\section{Patients and tissue specimens}

In this study, 208 paraffin-embedded LSCC tissue samples with previously untreated, surgically resectable, advanced-stage (Stages III and IV) LSCC were collected from Sun Yat-sen University Cancer Center (SYSUCC), Guangzhou, China. All patients were pathologically diagnosed and received surgery between May 1999 and December 2009. This study was approved by the Clinical Research Ethics Committee of SYSUCC.

\section{Immunohistochemistry}

We examined LKB1 and p16 expression in the 208 human LSCC tissue samples using immunohistochemistry. The paraffin-embedded sections (4-um thick) were heated at $60{ }^{\circ} \mathrm{C}$ for 2 hours and deparaffinized in xylene. Antigen retrieval was performed using EDTA (PH 8.0) in high-pressure steam four 5 minutes. Sections were incubated with $1 \%$ bovine serum Albumin to block nonspecific binding, and incubated with anti-p16 antibody (1:100 dilution; JC8, Santa Cruz, CA), antibody to LKB1 (1:200 dilution; Cell Signaling Technologies), and then the slides were placed in a moist chamber overnight at $4^{\circ} \mathrm{C}$. After washing, the tissue sections were treated with biotinylated anti-rabbit secondary antibody (Abcam), and horseradish peroxidase was subsequently applied. Finally, Meyer's hematoxylin was used for nuclear counterstaining.

All immunostained slides were judged by two independent pathologists without knowledge of the clinicopathologic information. p16 status was categorized as positive when p16 staining was present. LKB1 expression in the cytoplasm was evaluated independently. The intensity of LKB1 staining was categorized as follows: negative staining $($ score $=0)$; weak staining $($ score $=1)$; moderate 
staining $($ score $=2$ ); or strong staining $($ score $=3$ ). The percentage of immunoreactive cells was also assessed. The percentage of cells expressing LKB1 was classified as: $0 \%($ score $=0) ;<10 \%($ score $=1) ; 10-50 \%$ $($ score $=2) ;>50 \%($ score $=3)$. The staining index $=$ intensity score $\times$ distribution score (to obtain values of $0,1,2,3,4,6$, or 9). In this study, the cutoff value for LKB1 expression was defined based on a receiver operating characteristic (ROC) curve. A score $>4$ was used to classify tumors with high expression, whereas a score $\leq 4$ was used to indicate low expression.

\section{Clinical data}

Before treatment, all patients underwent a physical examination, routine hematologic testing, laryngoscopy, a pathologic assessment of biopsy tissue, magnetic resonance imaging of the head and neck, a chest X-ray, abdominal ultrasound, and an entire body bone scan, with or without positron-emission tomography-computed tomography (PET/CT). Overall stage was classified according to the seventh edition of the Union for International Cancer Control staging system [23].

Medical records were collected retrospectively. Data on age, smoking status, alcohol consumption, familial history of cancer, tumor localization, pathologic type, histologic grade, overall stage, $\mathrm{T}$ category, $\mathrm{N}$ category, treatment, and survival and metastasis status were recorded. Patients haven't received any anticancer therapy, such as chemotherapy and radiotherapy, only total or partial laryngectomy were conducted when pathologically diagnosed.

\section{Follow-up and outcome}

Follow-up evaluations were performed every 3 months within the first 3 years, every 6 months for the following 2 years, and annually thereafter until death. The last follow-up was December 2016; the median follow-up time was 94.6 (range: 10.3-212.2) months. Overall survival (OS) was defined as the time in months from the date of surgery until death from any cause within the follow-up period. Distant metastasis-free survival (DMFS) was measured from date of surgery to metastasis, death, or the last follow-up.

\section{Statistical analysis}

All statistical analyses were conducted using SPSS (version 22.0; IBM Corporation, Armonk, NY, USA). The survival curves were plotted by the GraphPad Prism 7.0 Software. LKB1 scores were categorized into the high and low expression groups according to the optimal cutoff value determined by ROC curve analysis. Survival curves were calculated using the Kaplan-Meier method and compared using the log-rank test. Cox proportional hazards analysis was used for univariate and multivariate analyses to explore the effects of clinicopathologic variables, LKB1 and p16 expression on survival. All parameters found to be significant in the univariate analysis were entered in a multivariate survival analysis using the Cox regression model. A two-sided probability value $<0.05$ was considered statistically significant.

\section{Results}

\section{Patients and tissue samples}

With approval by the institutional review board, a total of 208 advanced-stage laryngeal carcinoma tissue samples were obtained from SYSUCC. Clinicopathological characteristics of these patients were presented in Table 1.97 of 208 (46.6\%) patients died and 41 patients suffered diastant metastasis during our follow-up.

Table 1. Summary of the clinicopathological features of the 208 patients with laryngeal carcinoma

\begin{tabular}{|c|c|}
\hline \multirow[t]{3}{*}{ Characteristic } & Total \\
\hline & $(\mathrm{No}, \%)$ \\
\hline & $208(100)$ \\
\hline \multicolumn{2}{|l|}{ Age (years) } \\
\hline$<59$ & $107(51.4)$ \\
\hline$\geq 59$ & $101(48.6)$ \\
\hline \multicolumn{2}{|l|}{ Smoking } \\
\hline Yes & $184(88.5)$ \\
\hline No & $24(11.5)$ \\
\hline \multicolumn{2}{|c|}{ Overall stage (7th edition) } \\
\hline III & $98(47.1)$ \\
\hline IV & $110(52.9)$ \\
\hline \multicolumn{2}{|l|}{$\mathrm{N}$ category } \\
\hline N0-1 & $163(78.4)$ \\
\hline N2-3 & $45(21.6)$ \\
\hline \multicolumn{2}{|l|}{ Localization } \\
\hline Supraglottic & $84(40.4)$ \\
\hline Glottic & $97(46.6)$ \\
\hline Subglottic & $9(4.3)$ \\
\hline Combination & $18(8.7)$ \\
\hline \multicolumn{2}{|c|}{ Histologic grade } \\
\hline Well & $73(35.1)$ \\
\hline Moderate & $101(48.6)$ \\
\hline Poor & $34(16.3)$ \\
\hline \multicolumn{2}{|c|}{ LKB1 expression } \\
\hline Low & $128(61.5)$ \\
\hline High & $80(38.5)$ \\
\hline \multicolumn{2}{|l|}{ p16 status } \\
\hline Negative & $160(76.9)$ \\
\hline Positive & $48(23.1)$ \\
\hline \multicolumn{2}{|c|}{ Survival outcomes } \\
\hline Death & $97(46.6)$ \\
\hline Alive & $111(53.4)$ \\
\hline \multicolumn{2}{|c|}{ Distant metastasis } \\
\hline Yes & $41(19.7)$ \\
\hline No & $167(80.3)$ \\
\hline
\end{tabular}

\section{Immunohistochemistry}

Overall, 80/208 (38.5\%) patients exhibited high expression in laryngeal tumour tissue, whereas 
$128 / 208(61.5 \%)$ patients exhibited low expression. Of the 208 laryngeal tumour tissue samples, $48(23.1 \%)$ were p16-positive and $160(76.9 \%)$ were p16-negative. The representative figures were shown in Figure 1.

\section{Decreased LKB I Expression and survival}

The 5-year OS and DMFS rates for the entire cohort were $69.6 \%$ and $81.4 \%$, respectively. The cumulative 5-year OS and DMFS rates for patients with low LKB1 expression were $65.7 \%$ and $79.5 \%$, respectively, compared with $75.6 \%$ and $86.1 \%$, respectively, for patients with high LKB1 expression (log-rank test; $P=0.041$; Figure $2 \mathrm{~A}$ and $P=0.028$; Figure 2B). The 5-year OS rates for patients with negative p16 were $72.2 \%$, compared with $60.6 \%$ for patients with positive p16 (log-rank test; $P=0.038$;

\section{p16 expression}

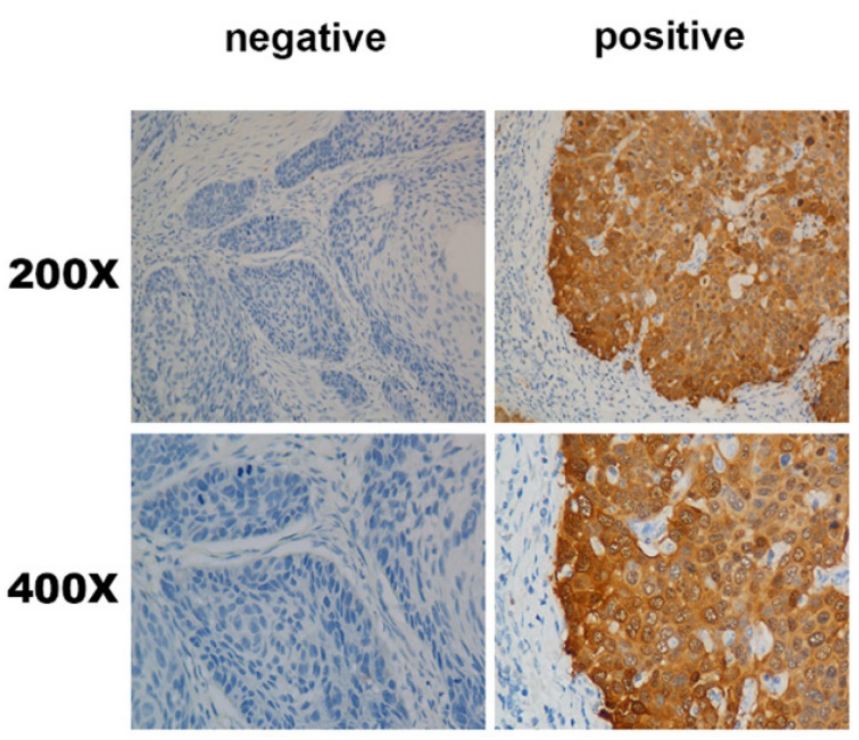

Figure 3A), but not for DMFS (log-rank test; $P=0.182$; Figure 3B).

\section{Cox proportional analyses of overall survival and distant metastasis-free survival}

Univariate Cox regression analysis demonstrated that the clinical variables age at diagnosis, albumin, overall stage, N-stage, LKB1 and p16 status were significantly associated with survival. A multivariate survival analysis indicated that high LKB1 expression was an independent prognostic factor for OS (hazard ratio [HR]: 1.628, 95\% confidence interval [CI]: 1.060-2.500, $P=0.026$; Table 2) and DMFS (HR: 2.182, 95\% CI: 1.069-4.456, $P=$ 0.032; Table not shown).

\section{LKB1 expression} low high

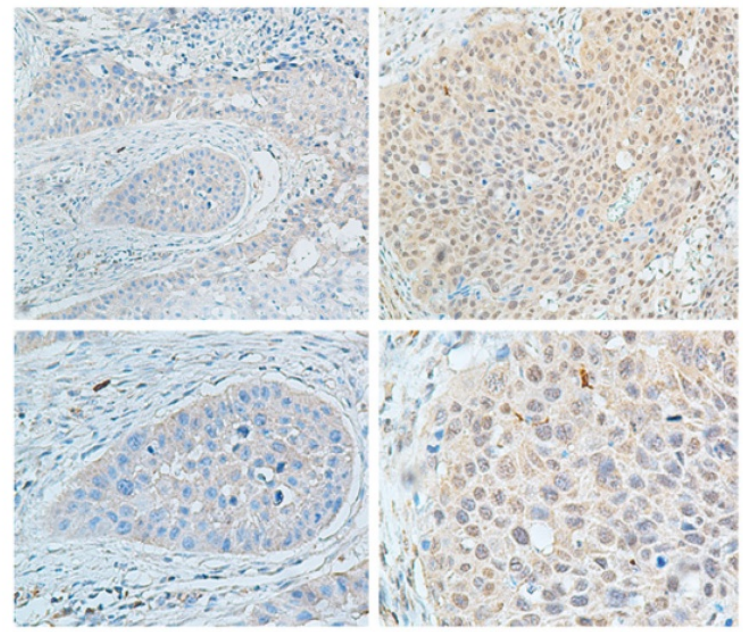

Figure 1. p16 and LKB1 overexpression in larynx tumors. Same region of same tumor staining positive for p16 (cytoplasm) and DNA-PKcs (cytoplasm), With magnification of $200 \times$ and $400 x$.
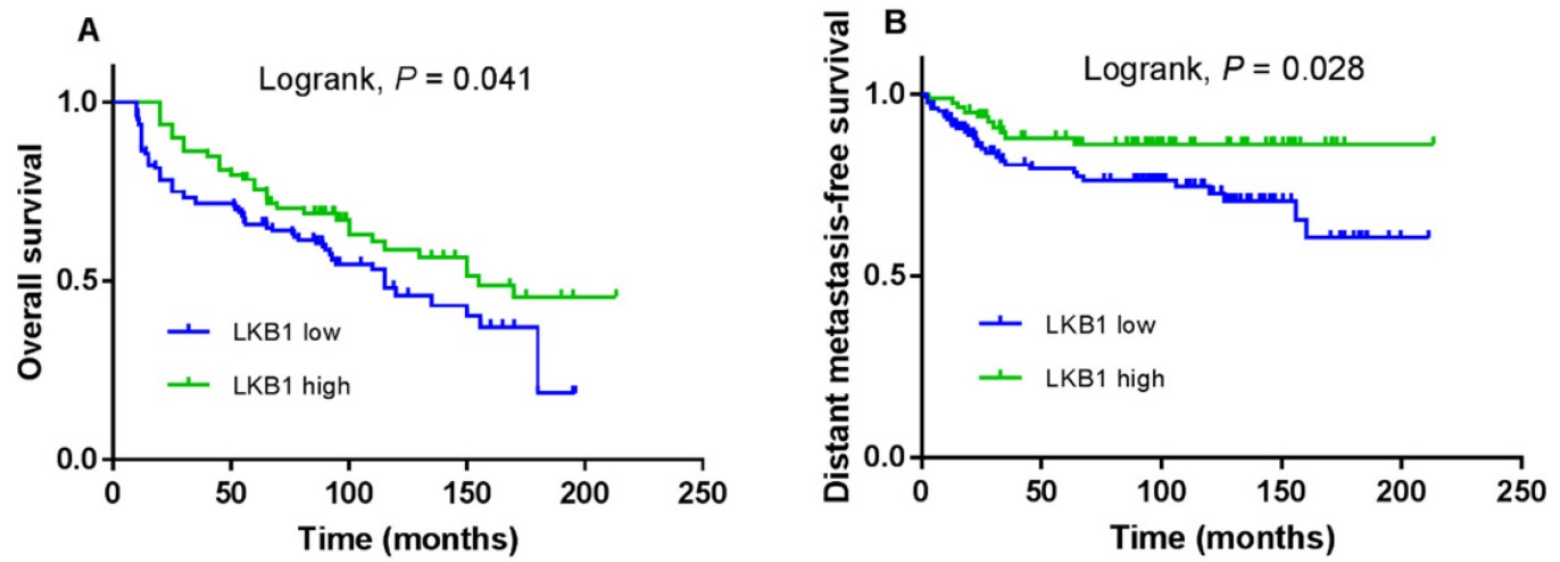

Figure 2. Kaplan-Meier overall survival (A) and distant metastasis-free survival (B) curves for 208 patients with laryngeal squamous cell carcinoma stratified by LKB1 expression. 

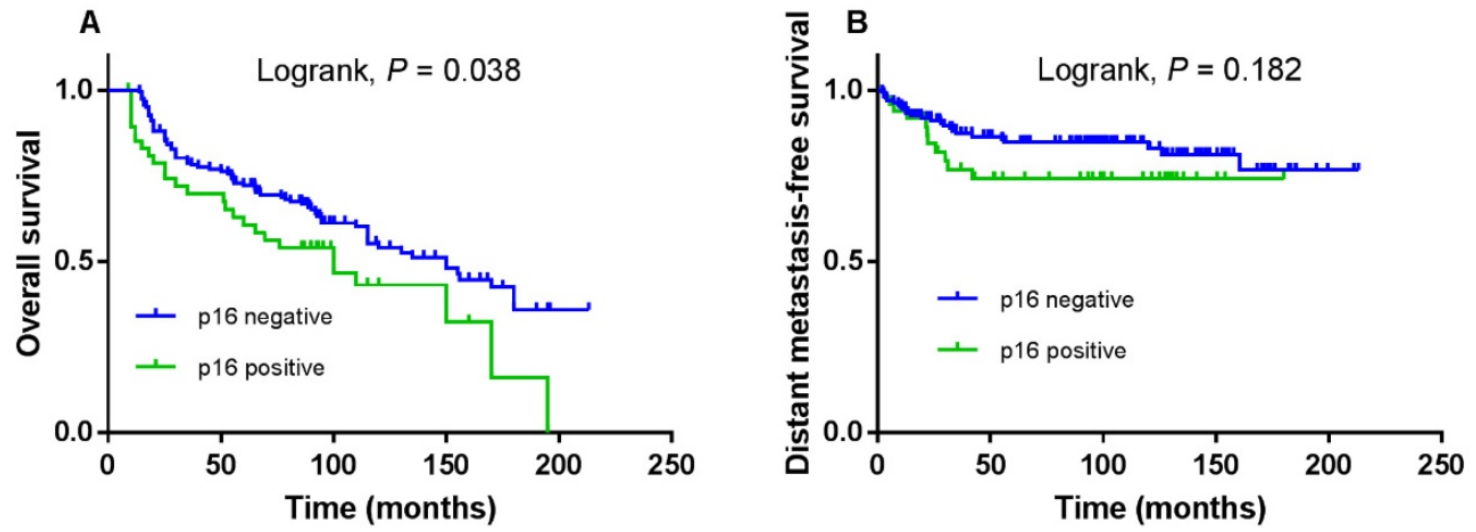

Figure 3. Kaplan-Meier overall survival (A) and distant metastasis-free survival (B) curves for 208 patients with laryngeal squamous cell carcinoma stratified by p16 expression.
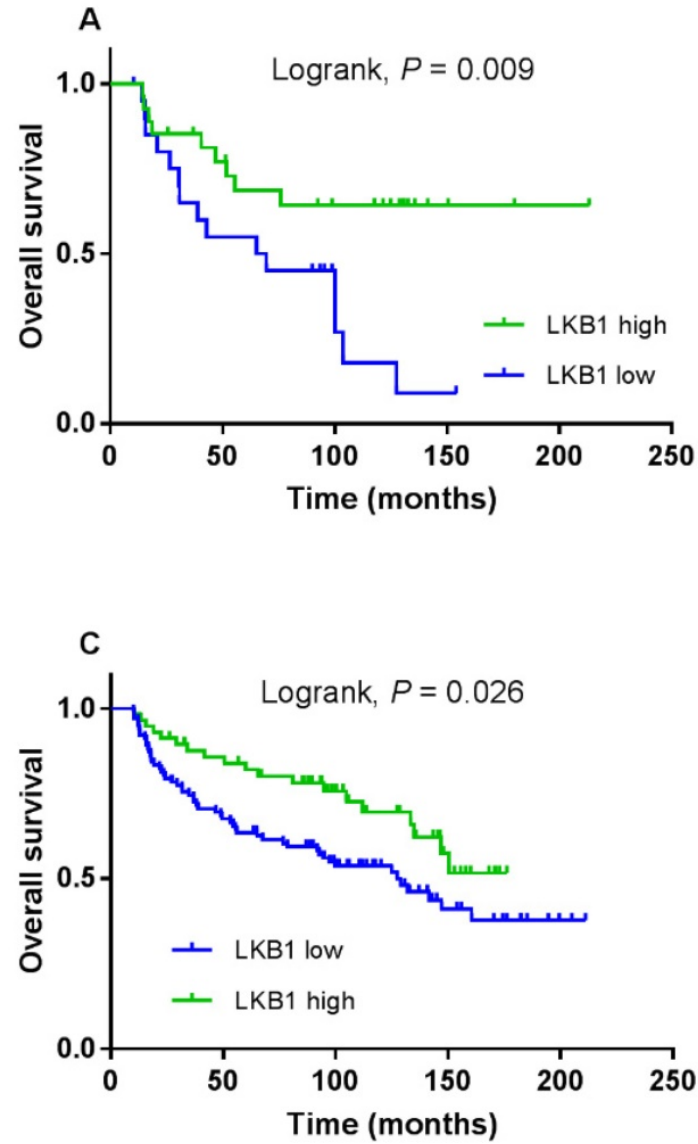
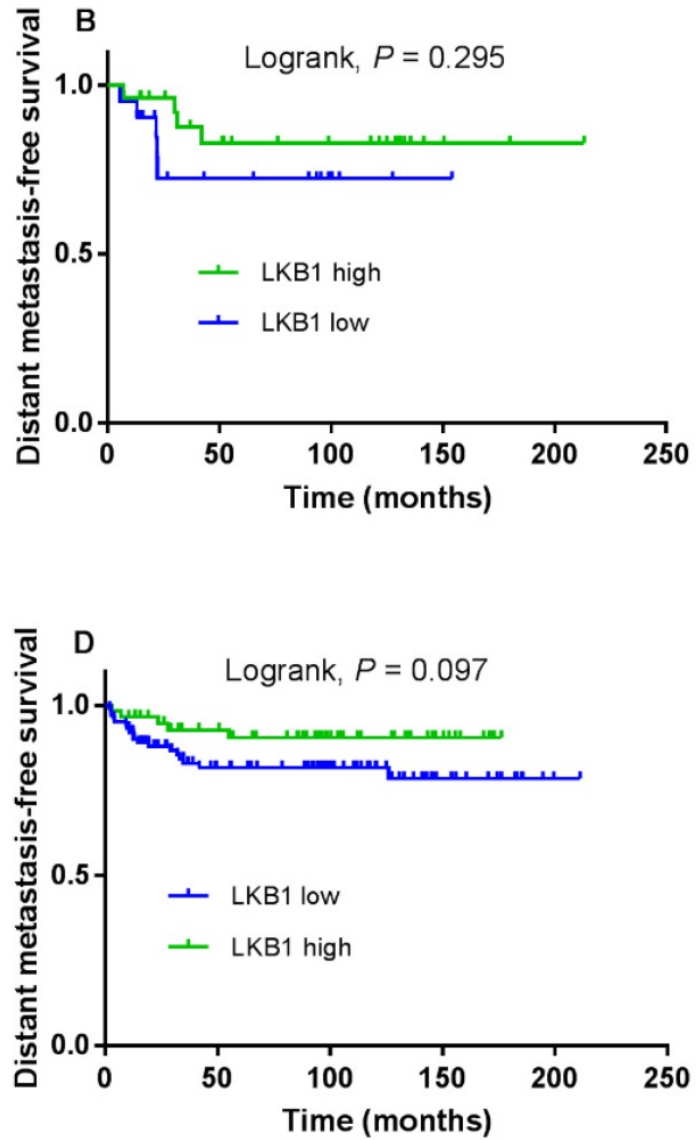

Figure 4. Kaplan-Meier overall survival (A) and distant metastasis-free survival (B) curves for patients with pl6 positive; overall survival (C) and distant metastasis-free survival (D) curves for patients with p16 negative.

\section{Subgroup analyses stratified by p16INK4a status}

Next, we stratified LKB1 expression by p16 status and evaluated its prognostic value. Compared with patients with low LKB1 expression, a trend toward higher OS was observed in patients with high LKB1 expression in the p16-positive and p16-negative subgroup (log-rank test; $P=0.009$; Figure $4 \mathrm{~A}$ and $P=$
0.026; Figure 4C). However, there was not the trend for DMFS in the two subgroups (log-rank test; $P=$ 0.295; Figure 4B and $P=0.097$; Figure 4D).

\section{Discussion}

The multivariate survival analysis revealed that decreased expression of LKB1 protein was significantly associated with OS (HR:1.628, 95\%CI: 
1.060-2.500, $P=0.026)$ and DMFS (HR: $2.182,95 \% \mathrm{CI}$ : $1.069-4.456, P=0.032$ ). Kaplan-Meier survival curves compared the 5-year OS and DMFS rates for patients with low and high LKB1 expression, the higher survival rates were received in the patients of high LKB1 expression group than the low group. Subgroup analyses were performed according to the p16 status and the OS benefit was also achieved.

Table 2. Cox's proportional hazards regression model of overall survival for the 208 patients with laryngeal carcinoma

\begin{tabular}{lllll}
\hline Variable & \multicolumn{2}{c}{ Univariate analysis } & \multicolumn{2}{c}{ Multivariate analysis } \\
\cline { 2 - 5 } & HR $(95 \% \mathrm{CI})$ & $P$ & HR $(95 \% \mathrm{CI})$ & $P$ \\
\hline $\begin{array}{l}\text { Age at diagnosis } \\
\text { (years) }\end{array}$ & & & & \\
$\geq 59$ vs. $<59$ & $1.522(1.018-2.275)$ & 0.041 & $1.642(1.092-2.470)$ & 0.017 \\
$\begin{array}{l}\text { Albumin }(\mathrm{g} / \mathrm{L}) \\
<42.5 \text { vs. } \geq 42.5\end{array}$ & $1.551(1.036-2.321)$ & 0.033 & & \\
$\begin{array}{l}\text { Overall stage } \\
\text { IV vs. III }\end{array}$ & $1.525(1.014-2.293)$ & 0.043 & \\
N category & $1.825(1.158-2.877)$ & 0.010 & \\
$\begin{array}{l}\text { N2-3 vs. N0-1 } \\
\text { LKB1 expression }\end{array}$ & $1.541(1.011-2.348)$ & 0.044 & $1.628(1.060-2.500)$ & 0.026 \\
$\begin{array}{l}\text { Low vs. high } \\
\text { p16 status }\end{array}$ & $1.591(1.019-2.482)$ & 0.041 & \\
Positive vs. negative & & &
\end{tabular}

LKB1, as a more recognized tumor suppressor gene, has been widely studied. Wei $C$ et al. even found that mutations of P53 and LKB1 gene cooperate in the acceleration of tumorigenesis [24]. While, the exact molecular mechanism of tumor suppression is not yet distinguished. Studies have revealed LKB1 interacts with some downstream signalings such as LKB1-AMPK [25], MARK/Par-1 [26], SIK [27], SNRK [28] among other pathways, the activations of them can subsequently inhibit anabolism and induce cell cycle arrest, and finally suppress cancer cell growth. Research has also found that LKB1 can not only suppress cancer cell growth or induce cancer cell death, but also can inhibit cancer cell metastasis. Overexpression of LKB1 can significantly inhibit the invasion and metastasis of cancer cells, in breast cancer, LKB1 expression decreases the microvessel density, and lung metastasis [20], and LKB1 loss promote lymph node metastasis in head and neck squamous cell carcinoma [29]. It has been reported in the cervical cancer cells [30], LKB1 overexpression can inhibit its growth, with a complete signal pathway of cervical cancer cell lines have an inhibitory effect. However, the study of molecular mechanisms of cancer still exist many unknown, control more downstream genes? Is there a new pathway involved in its anti-cancer mechanism? The molecular mechanism of LKB1 will help to find more critical new ideas and targets for the treatment of cancer with more in-depth explorations. Based on the potential role in the development of several cancer. In our study, compare to the low expression group, the patients with high expression LKB1 obtain obviously longer OS and DMFS. The function of the signal pathway in the invasion and migration of cancer cells and therapeutic strategies aimed at those pathways in tumors should also be further studied.

A study [31] have recently demonstrated that LKB1 is a crucial regulator of genome integrity, lacking LKB1 increases sensitivity to irradiation, accumulates more DNA double-strand breaks, and further lead to an increased mutation rate, compared with that of high LKB1 expressing. The results identify the therapeutic implications of LKB1 in mediating the DNA damage response and repair, also suggest that the LKB1 may be as a target for cancer prevention. LKB1 can inhibit cancer cell growth with many mechanisms. In line with LKB1, AMPK also plays a central role in the regulation of whole-body energy metabolism, LKB1 induce cell cycle arrest of cancer cells through AMPK signalling [32]. In addition, under the condition of hypoxia, which influences the environment of tumour, LKB1 may inhibit cancer cells growth through regulation of hypoxia inducible factor 1 (HIF-1) [33]. Hypoxia is a negative prognostic and predictive factor owing to its multiple contributions to resistance to therapy from the aspects of chemoresistance, radioresistance, angiogenesis, vasculogenesis, invasiveness, metastasis, resistance to cell death, altered metabolism and genomic instability [34]. However, despite the existing information on all the signalling mechanisms. Here, it is a long-standing goal of exploiting the approaches for linking LKB1 and signalling, therapeutic resistance together and further investigates the therapy for cancers.

\section{Conclusions}

In summary, our results may indicate that LKB1 plays a role in tumour invasion and metastasis. Decreased expression of the LKB1 protein in human laryngeal carcinoma is significantly associated with shorter overall survival and distant metastasis-free survival. While, several related pathways of survival and metastasis have not been identified clearly and applied to the clinical decision. Furthermore, there is a necessity to explore the functions of LKB1 in cellular and molecular levels. Ultimately, an understanding of LKB1 function and how a compromised LKB1 pathway impacts metastasis could reveal new opportunities for predicting and controlling LSCC metastasis. 


\section{Acknowledgments}

This work was supported by grants from the Medical Research Fundation of Sun Yat - sen University of Guangdong Province, China [grant number A2016011], the Natural Science Foundation of Guangdong Province, China [grant number 2016A020215083] and Zhejiang Province Provincial Public Welfare Technology Application Research Plan, China [grant number 2014C33196]. The funding agency had no role in the study design, data collection and analysis, decision to publish, or preparation of the manuscript.

\section{Competing Interests}

The authors have declared that no competing interest exists.

\section{References}

1. Jenne DE, Reimann H, Nezu J, Friedel W, Loff S, Jeschke R, et al. Peutz-Jeghers syndrome is caused by mutations in a novel serine threonine kinase. Nature genetics. 1998; 18: 38-43.

2. Shackelford DB, Shaw RJ. The LKB1-AMPK pathway: metabolism and growth control in tumour suppression. Nature reviews Cancer. 2009; 9: 563-75.

3. van Lier MG, Wagner A, Mathus-Vliegen EM, Kuipers EJ, Steyerberg EW, van Leerdam ME. High cancer risk in Peutz-Jeghers syndrome: a systematic review and surveillance recommendations. The American journal of gastroenterology. 2010; 105: 1258-64

4. Latchford AR, Phillips RK. Gastrointestinal polyps and cancer in Peutz-Jeghers syndrome: clinical aspects. Familial cancer. 2011; 10: 455-61.

5. Hemminki A. The molecular basis and clinical aspects of Peutz-Jeghers syndrome. Cellular and molecular life sciences : CMLS. 1999; 55: 735-50.

6. Kottakis F, Nicolay BN, Roumane A, Karnik R, Gu H, Nagle JM, et al. LKB1 loss links serine metabolism to DNA methylation and tumorigenesis. Nature. 2016; 539: 390-5.

7. Hawley SA, Boudeau J, Reid JL, Mustard KJ, Udd L, Makela TP, et al Complexes between the LKB1 tumor suppressor, STRAD alpha/beta and $\mathrm{MO} 25$ alpha/beta are upstream kinases in the AMP-activated protein kinase cascade. Journal of biology. 2003: $2 \cdot 28$.

8. Woods A, Johnstone SR, Dickerson K, Leiper FC, Fryer LG, Neumann D, et al. LKB1 is the upstream kinase in the AMP-activated protein kinase cascade. Current biology : CB. 2003; 13: 2004-8.

9. Xiao B, Sanders MJ, Carmena D, Bright NJ, Haire LF, Underwood E, et al. Structural basis of AMPK regulation by small molecule activators. Nature communications. 2013; 4: 3017.

10. $\mathrm{Li} \mathrm{L}, \mathrm{Li} \mathrm{W}$. Epithelial-mesenchymal transition in human cancer: comprehensive reprogramming of metabolism, epigenetics, and differentiation. Pharmacology \& therapeutics. 2015; 150: 33-46.

11. Larue L, Bellacosa A. Epithelial-mesenchymal transition in development and cancer: role of phosphatidylinositol 3' kinase/AKT pathways. Oncogene. 2005; 24: 7443-54.

12. Yang J, Weinberg RA. Epithelial-mesenchymal transition: at the crossroads of development and tumor metastasis. Developmental cell. 2008; 14: 818-29.

13. He TY, Tsai LH, Huang CC, Chou MC, Lee H. LKB1 loss at transcriptional level promotes tumor malignancy and poor patient outcomes in colorectal cancer. Annals of surgical oncology. 2014; 21 Suppl 4: S703-10.

14. Jiang L, Liang X, Liu M, Wang W, Ma J, Guo Q, et al. Reduced expression of liver kinase B1 and Beclin1 is associated with the poor survival of patients with non-small cell lung cancer. Oncology reports. 2014; 32: 1931-8.

15. Li J, Liu J, Li P, Mao X, Li W, Yang J, et al. Loss of LKB1 disrupts breast epithelial cell polarity and promotes breast cancer metastasis and invasion. Journal of experimental \& clinical cancer research : CR. 2014; 33: 70.

16. Kim CJ, Cho YG, Park JY, Kim TY, Lee JH, Kim HS, et al. Genetic analysis of the LKB1/STK11 gene in hepatocellular carcinomas. European journal of cancer (Oxford, England : 1990). 2004; 40: 136-41.

17. Qiu W, Schonleben F, Thaker HM, Goggins M, Su GH. A novel mutation of STK11/LKB1 gene leads to the loss of cell growth inhibition in head and neck squamous cell carcinoma. Oncogene. 2006; 25: 2937-42.

18. Xiao J, Zou Y, Chen X, Gao Y, Xie M, Lu X, et al. The Prognostic Value of Decreased LKB1 in Solid Tumors: A Meta-Analysis. PloS one 2016; 11: e0152674.

19. Fenton H, Carlile B, Montgomery EA, Carraway H, Herman J, Sahin F, et al. LKB1 protein expression in human breast cancer. Applied immunohistochemistry \& molecular morphology : AIMM. 2006; 14: 146-53.
20. Zhuang ZG, Di GH, Shen ZZ, Ding J, Shao ZM. Enhanced expression of LKB1 in breast cancer cells attenuates angiogenesis, invasion, and metastatic potential. Molecular cancer research : MCR. 2006; 4: 843-9.

21. Zhang HS, Postigo AA, Dean DC. Active transcriptional repression by the $\mathrm{Rb}-\mathrm{E} 2 \mathrm{~F}$ complex mediates G1 arrest triggered by p16INK4a, TGFbeta, and contact inhibition. Cell. 1999; 97: 53-61.

22. von Knebel Doeberitz M. New markers for cervical dysplasia to visualise the genomic chaos created by aberrant oncogenic papillomavirus infections. European journal of cancer (Oxford, England : 1990). 2002; 38: 2229-42.

23. Edge SB, Compton CC. The American Joint Committee on Cancer: the 7th edition of the AJCC cancer staging manual and the future of TNM. Annals of surgical oncology. 2010; 17: 1471-4.

24. Wei C, Amos CI, Stephens LC, Campos I, Deng JM, Behringer RR, et al. Mutation of Lkb1 and p53 genes exert a cooperative effect on tumorigenesis. Cancer research. 2005; 65: 11297-303.

25. Shaw RJ, Kosmatka M, Bardeesy N, Hurley RL, Witters LA, DePinho RA, et al. The tumor suppressor LKB1 kinase directly activates AMP-activated kinase and regulates apoptosis in response to energy stress. Proceedings of the National Academy of Sciences of the United States of America. 2004; 101: 3329-35.

26. Wang JW, Imai Y, Lu B. Activation of PAR-1 kinase and stimulation of tau phosphorylation by diverse signals require the tumor suppressor protein LKB1. The Journal of neuroscience : the official journal of the Society for Neuroscience. 2007; 27: 574-81.

27. Hashimoto $Y K$, Satoh $T$, Okamoto $M$, Takemori $H$. Importance of autophosphorylation at Ser186 in the A-loop of salt inducible kinase 1 for its sustained kinase activity. Journal of cellular biochemistry. 2008; 104: 1724-39.

28. Jaleel M, McBride A, Lizcano JM, Deak M, Toth R, Morrice NA, et al Identification of the sucrose non-fermenting related kinase SNRK, as a novel LKB1 substrate. FEBS letters. 2005; 579: 1417-23.

29. Kline ER, Muller S, Pan L, Tighiouart M, Chen ZG, Marcus AI Localization-specific LKB1 loss in head and neck squamous cell carcinoma metastasis. Head \& neck. 2011; 33: 1501-12.

30. Zhang X, Chen H, Wang X, Zhao W, Chen JJ. Expression and transcriptional profiling of the LKB1 tumor suppressor in cervical cancer cells. Gynecologic oncology. 2014; 134: 372-8.

31. Gupta R, Liu AY, Glazer PM, Wajapeyee N. LKB1 preserves genome integrity by stimulating BRCA1 expression. Nucleic acids research. 2015; 43: 259-71.

32. Li N, Huang D, Lu N, Luo L. Role of the LKB1/AMPK pathway in tumor invasion and metastasis of cancer cells (Review). Oncology reports. 2015; 34: 2821-6.

33. Shackelford DB, Vasquez DS, Corbeil J, Wu S, Leblanc M, Wu CL, et al. mTOR and HIF-1alpha-mediated tumor metabolism in an LKB1 mouse model of Peutz-Jeghers syndrome. Proceedings of the National Academy of Sciences of the United States of America. 2009; 106: 11137-42.

34. Wilson WR, Hay MP. Targeting hypoxia in cancer therapy. Nature reviews Cancer. 2011; 11: 393-410. 